\title{
Polynomial Type Padé Approximants
}

\author{
By Géza Németh and Magda Zimányi
}

\begin{abstract}
Some results are established giving conditions on $f(x)$ so that its main diagonal Padé approximation $R_{n}(x)$ is of the form $P_{n}(x) / P_{n}(-x)$, where $P_{n}(x)$ is a polynomial in $x$ of degree $n$. A number of applications to special functions are presented. Numerical computations are given for the gamma function using the "bignum" arithmetical facilities of formula manipulation languages REDUCE2, FORMAC.
\end{abstract}

1. Introduction. Suppose $f(x)$ is represented by the at least formal series

$$
f(x)=\sum_{k=0}^{\infty} c_{k} x^{k},
$$

then the main diagonal Padé approximation to $f(x)$ is of the form

$$
R_{n}(x)=P_{n}(x) / Q_{n}(x),
$$

where $P_{n}(x)$ and $Q_{n}(x)$ are polynomials in $x$ of degree $n$. That is,

$$
f(x)-R_{n}(x)=O\left(x^{2 n+1}\right) .
$$

If

$$
Q_{n}(x)=P_{n}(-x), \quad R_{n}(x)=P_{n}(x) / P_{n}(-x),
$$

then evaluation of $R_{n}(x)$ can be considerably simplified in the sense that the number of operations required is as though only a single polynomial needs to be evaluated instead of two polynomials. For a case in point, it is known (e.g., [7]) that if $f(x)=e^{-x}$, then (4) is true. The simplification results by separately evaluating the odd and even parts of $P_{n}(x)$. In this connection, see the discussion given by Luke [4, p. 192] and [5, p. 48].

In this paper, we present some more cases where the Pade approximant is of the form (4). We call such rational approximations polynomial type Padé approximants.

2. Functions with Polynomial Type Padé Approximants. We prove

THEOREM 1. Let $f(x)$ be given by (1), and suppose that (2) and (3) hold. Further, suppose that

$$
f(x) \cdot f(-x)=1
$$

Then (4) is true.

Received May 12, 1981; revised August 19, 1981.

1980 Mathematics Subject Classification. Primary 41A20, 41A21, 68C20; Secondary 33A15, 33-04.

Key words and phrases. Rational approximation, Padé approximation, Padé approximants, special functions, gamma function, symbolic computing, REDUCE2, FORMAC. 
Note 1. It is not difficult to see that (5) is equivalent to the condition

$$
\ln f(x)=\sum_{k=1}^{\infty} g_{k} x^{2 k-1} \text {. }
$$

That is, the formal expansion of the logarithm of the function contains only odd powers of $x$.

Note 2. This result first appeared in [6].

Proof. It is known that if $R_{n}(x)$ as given by (2) is the Padé approximation of $f(x)$, then the reciprocal of (2) is the Padé approximation of $1 / f(x)$; see [2]. So

$$
\frac{Q_{n}(x)}{P_{n}(x)}=\frac{P_{n}(-x)}{Q_{n}(-x)} .
$$

That is $Q_{n}(x)=\rho P_{n}(-x)$ and $P_{n}(x)=\rho Q_{n}(-x)$, where $\rho$ is a constant. Since $Q_{n}(0)=P_{n}(0)=c_{0}, \rho=1$ and $(4)$ holds.

An interesting application of our result is the Stirling series. It is a well-known fact that

$$
\Gamma(x)=x^{x} e^{-x} \sqrt{\frac{2 \pi}{x}} \cdot F(x)
$$

where

$$
F(x) \sim 1+\frac{1}{12 x}-\frac{1}{288 x^{2}}-\frac{1}{51840 x^{3}}+\cdots .
$$

But we also know that

$$
\ln F(x) \sim \sum_{k=1}^{\infty} f_{k} x^{-2 k-1},
$$

where $f_{k}=B_{2 k} / 2 k(2 k-1)$ and $B_{k}$ are the Bernoulli numbers. Thus by Theorem 1 we get that the Padé approximants of $F(x)$ are of type (4). Thus

$$
F_{1}(x)=\frac{24 x+1}{24 x-1}, \quad F_{2}(x)=\frac{8640 x^{2}+360 x+293}{8640 x^{2}-360 x+293} .
$$

The next two numerator polynomials of $F_{3}(x)$ and $F_{4}(x)$ in integer coefficients are

$$
\begin{gathered}
P_{3}(x)=425295360 x^{3}+17720640 x^{2}+120170160 x+4406147 \\
P_{4}(x)=153494651842560 x^{4}+6395610493440 x^{3}+124448535691200 x^{2} \\
+4968467473872 x+2749505046083
\end{gathered}
$$

In Section 3 we shall present the integer coefficients of the polynomials $P_{n}(x)$ for $n=1, \ldots, 5$, and in the tables presented at the end of this paper for $n=1, \ldots, 10$.

For another application, consider

$$
G(x)=\frac{\Gamma\left(x+\frac{1}{2}\right)}{\Gamma(x+1)} .
$$

In this instance

$$
G(x) \sim x^{-1 / 2}\left(1-\frac{1}{8 x}+\frac{1}{128 x^{2}}+\frac{5}{1024 x^{3}}+\cdots\right) .
$$


Put

$$
z=\frac{1}{x}, \quad x G(x)=H(z) .
$$

Then $H(z)$ is of type (6). So

$$
H(z) \sim x^{1 / 2} \frac{S_{n}(z)}{S_{n}(-z)}
$$

$$
\begin{aligned}
& S_{1}(z)=16-z \\
& S_{2}(z)=256-16 z+11 z^{2} \\
& S_{3}(z)=45056-2816 z+13280 z^{2}-709 z^{3} \\
& S_{4}(z)=46465024-2904064 z+38648064 z^{2}-2290720 z^{3}+1072189 z^{4}
\end{aligned}
$$

If we take $z=k, k$ an integer, we get a remarkable approximation to the asymptotic series in the Wallis formula for large $k$ :

$$
\frac{1 \cdot 3 \ldots(2 k-1)}{1 \cdot 2 \ldots 2 k} \sim \frac{1}{\sqrt{\pi k}} \frac{S_{n}(k)}{S_{n}(-k)} .
$$

The third example is the binomial function $((1-x) /(1+x))^{\circ}$. This function satisfies condition (6). So Padé approximants exist of the form

$$
\left(\frac{1-x}{1+x}\right)^{\sigma} \sim \frac{D_{n}(-x)}{D_{n}(x)}, \quad n=0,1,2, \ldots,
$$

where

$$
\begin{aligned}
& D_{0}(x)=1 \\
& D_{1}(x)=1+\sigma x \\
& D_{2}(x)=1+\sigma x-\frac{1-\sigma^{2}}{3} x^{2} \\
& D_{3}(x)=1+\sigma x-\frac{3-2 \sigma^{2}}{5} x^{2}-\frac{\sigma}{15}\left(4-\sigma^{2}\right) x^{3} \\
& D_{4}(x)=1+\sigma x-\frac{6-3 \sigma^{2}}{7} x^{2}-\frac{\left(11-2 \sigma^{2}\right)}{21} x^{3}+\frac{\left(1-\sigma^{2}\right)\left(9-\sigma^{2}\right)}{105} x^{4} .
\end{aligned}
$$

The polynomials $D_{n}(x)$ satisfy the recurrence relation

$$
D_{n+1}(x)=D_{n}(x)-\frac{n^{2}-\sigma^{2}}{4 n^{2}-1} x^{2} D_{n-1}(x), \quad n=1,2,3, \ldots
$$

The function $((1-i x) /(1+i x))^{i \nu}=\exp \{-2 \nu \arctan x\}$ can be approximated in a similar manner. The Padé approximants also exist and are of the form

$$
\left(\frac{1-i x}{1+i x}\right)^{i v} \sim \frac{C_{n}(-x)}{C_{n}(x)}
$$




$$
\begin{aligned}
& C_{0}(x)=1, \\
& C_{1}(x)=1-\nu x, \\
& C_{2}(x)=1-\nu x+\frac{1+\nu^{2}}{2} x^{2}, \\
& C_{3}(x)=1-\nu x+\frac{3+2 \nu^{2}}{5} x^{2}-\frac{\nu}{15}\left(4+\nu^{2}\right) x^{3}, \\
& C_{4}(x)=1-\nu x+\frac{6+3 \nu^{2}}{7} x^{2}-\frac{\nu}{21}\left(11+2 \nu^{2}\right) x^{3}+\frac{\left(1+\nu^{2}\right)\left(9+\nu^{2}\right)}{105} x^{4} .
\end{aligned}
$$

The polynomials $C_{n}(x)$ satisfy the recurrence relation

$$
C_{n+1}(x)=C_{n}(x)+\frac{n^{2}+\nu^{2}}{4 n^{2}-1} x^{2} C_{n-1}(x), \quad n=1,2,3, \ldots
$$

Another result is

THEOREM 2. Let $f(x, a)$ be given by (1) and suppose that (2) and (3) hold where $P_{n}(x)$ is replaced by $P_{n}(x, a)$, etc. Further, suppose that

$$
f(x, a) f(x,-a)=1 \text {. }
$$

Then

$$
R_{n}(x, a)=\frac{P_{n}(x, a)}{Q_{n}(x, a)}, \quad Q_{n}(x, a)=P_{n}(x,-a)
$$

The proof is much like that for Theorem 1, and we omit details.

For an illustration of this theorem, we have

$$
(1+x)^{\alpha}=\frac{P_{n}(x, \alpha)}{P_{n}(x,-\alpha)}-\frac{\alpha \frac{(1-\alpha)_{n}(1+\alpha)_{n}}{2 n !} \int_{0}^{x} \frac{(x-t)^{n} t^{n}}{(1+t)^{n+1-\alpha}} d t}{P_{n}(x,-\alpha)}
$$

where

$$
P_{n}(x, \alpha)={ }_{2} F_{1}(-n,-n-\alpha ;-2 n ;-x),
$$

a result given by Luke in $[4$, p. 170].

3. Numerical Results. We have computed the coefficients of the polynomial type Padé approximants for $F(x)$ in Stirling's formula for $\Gamma(z)$ in (8).

If the asymptotic series of $F(x)$ is

$$
F(x) \sim \sum_{i=0}^{\infty} c_{i} x^{-i}
$$

the $p_{i}^{(n)}$ and $q_{i}^{(n)}$ coefficients of the Padé approximation of $F(x)$,

$$
F(x) \sim \frac{P_{n}(x)}{Q_{n}(x)}=\frac{P_{n}(x)}{P_{n}(-x)},
$$


can be computed from the equations

$$
\sum_{i=1}^{n} c_{n+k-i} q_{i}^{(n)}=-c_{n+k}, \quad k=1,2, \ldots, n,
$$

and

$$
p_{i}^{(n)}=\sum_{k=0}^{i} q_{k}^{(n)} c_{i-k}, \quad q_{0}^{(n)}=1, i=0,1, \ldots, n .
$$

Because in our case $p_{i}^{(n)}=(-1)^{i} q_{i}^{(n)}$, Eqs. (31) are used only for checking the correctness of the computation of $q_{i}^{(n)}$ by (30).

Equation (30) can be solved for $q_{i}^{(n)}$ by a simplified version of Trench's method [9].

For $n=0$

$$
q_{0}^{(0)}=1, \quad \lambda_{0}=1
$$

For $n>0$

$$
\begin{gathered}
\lambda_{n}=\sum_{i=0}^{n-1} c_{n+i} q_{n-1-i}^{(n-1)}, \quad n=1,2, \ldots, \\
q_{0}^{(n)}=1, \quad q_{1}^{(n)}=-\frac{1}{24}, \\
q_{i}^{(n)}=q_{i}^{(n-1)}-\frac{\lambda_{n}}{\lambda_{n-1}} q_{i-2}^{(n-2)}, \quad \text { for } i=2,3, \ldots, n, n=2,3, \ldots,
\end{gathered}
$$

where

$$
\begin{aligned}
q_{n+1}^{(n)} & =0, \\
q_{j}^{(k)} & =0, \quad \text { if } j<0 \text { or } k<0 .
\end{aligned}
$$

In the numerical computation of the Pade coefficients we have made use of the fact that algebraic manipulation languages offer a facility for calculation with large integer and rational numbers ("bignum" arithmetics).

The $p_{i}^{(n)}$ rational coefficients were computed from Eqs. (32) and (33). The Padé approximant was then taken in the form

$$
F(x) \sim \frac{P_{n}(x)}{P_{n}(-x)}=\frac{P_{n}^{*}(x)}{P_{n}^{*}(-x)},
$$

where $P_{n}^{*}(x)$ has integer coefficients, and we computed the $p_{i}^{*(n)}$ 's by multiplying the $p_{i}^{(n)}$ 's by their common denominators.

The numerical values of the $c_{i}$ coefficients of the asymptotic series of $F(z)$, for $n=1, \ldots, 20$, were taken from the paper by J. W. Wrench [10].

The $p_{i}^{*(n)}$ integer coefficients are listed in Table 1 for $n=1, \ldots, 5$, and in the tables presented at the end of this paper for $n=1, \ldots, 10$. 


\section{TABLE 1}

Integer coefficients of the polynomial type Padé approximants

$$
\text { for } \Gamma(z)(n=1,2, \ldots, 5)
$$

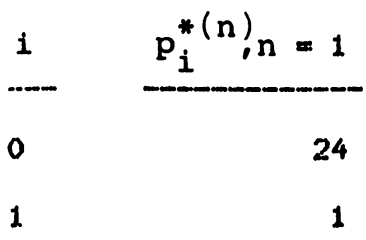

$\begin{array}{lr}i & p_{i}^{*(n)}, n=2 \\ 0 & 8640 \\ 1 & 360 \\ 2 & 293\end{array}$

$i \quad p_{i}^{*(n)}, n=3$

425295360

$1 \quad 17720640$

2120170160

$3 \quad 4406147$

i $\quad p_{i}^{*(n)}, n=4$

$0 \quad 153494651842560$

$1 \quad 6395610493440$

2124448535691200

$3 \quad 4968467473872$

$4 \quad 2749505046083$

i $\quad \mathrm{p}_{i}^{*(\mathrm{n})}, \mathrm{n}=5$

1137902961267501753139200

47412623386145906380800

$2 \quad 2067139141929439679969280$

$3 \quad 84522938940099251645760$

$4 \quad 3437 \quad 874252283265242 \quad 23240$

$5 \quad 11857916887903760599781$ 
The programming was done in the REDUCE2 language for symbolic computing [3]. We have reprogrammed the algorithm in PL/I-FORMAC [1], [8] too and obtained the same results. Both versions computed the $p_{i}^{*(n)}$ coefficients recursively from (32) and (33).

A possibility was provided by the REDUCE2 facility for matrix calculations to do a further check by solving the linear equations (30).

The computation was carried out on the ES-1040 computer of the Central Research Institute for Physics, Budapest. (The ES-1040 has a 1 MByte memory and a processing speed of $\sim 380$ thousand instruction $/ \mathrm{sec}$.)

The computation of the $p_{i}^{*(n)}$ coefficients took about 10 minutes of central processing unit time.

Acknowledgements. The authors are indebted to Professor A. C. Hearn (RAND Corporation, Santa Monica, California, U.S.A.) and to H. W. Homrighausen (Kernforschungsanlage Jülich, Federal Republic of Germany) for making the REDUCE2 resp. FORMAC73 systems available to us.

We are also grateful to Professor Hearn and Professor J. Fitch (University of Bath, Great Britain) for their help to correct an error in the "bignum" arithmetics package of the Utah LISP interpreter used by the REDUCE/360 system.

Hungarian Academy of Sciences

Central Research Institute for Physics

H-1525 Budapest, P.O.B. 49

Hungary

1. K. BAHR, "Utilizing the FORMAC novelties," SIGSAM Bull. No. 33, 1975, pp. 21-24.

2. G. A. BAKer, JR., Essentials of Padé Approximants, Academic Press, New York, 1975.

3. A. C. HEARN, REDUCE2 User's Manual, UCP-19, University of Utah, Salt Lake City, Utah, 1973.

4. Y. L. LukE, The Special Functions and Their Approximations, Vol. 2, Academic Press, New York and London, 1969.

5. Y. L. LUKE, Mathematical Functions and Their Approximations, Academic Press, New York and London, 1975.

6. K. NEMETH, Padé-Type Approximations (in Hungarian), Unpublished diploma work at Eötvös Loránd University, Budapest, 1980.

7. O. Perron, Die Lehre von den Kettenbrüchen. Vols. I, II, Teubner, Stuttgart, 1954.

8. R. TOBEY, J. BAKER, R. CREWS, P. MARKS \& K. Victor, PL/I-FORMAC interpreter, 1967.

9. W. F. TrenCh, "An algorithm for the inversion of finite Hankel matrices," SIAM J. Appl. Math., v. 13, 1965, pp. 1102-1107.

10. J. W. WrenCH, JR., "Concerning two series for the gamma function,” Math. Comp., v. 22, 1968, pp. 617-626.

Integer coefficients of the polynomial type Padé

approximants for $\Gamma(z)(n=1,2, \ldots, 10)$

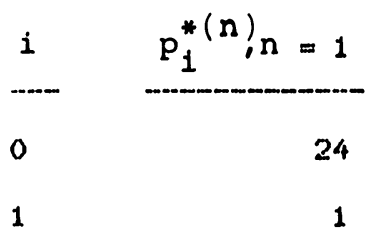




$\begin{array}{rr}i & p_{i}^{*(n)}, n=2 \\ 0 & 8640 \\ 1 & 360 \\ 2 & 293\end{array}$

i $\quad p_{i}^{*(n)}, n=3$

$0 \quad 425295360$

$1 \quad 17720640$

$2 \quad 120170160$

$3 \quad 4406147$

i $\quad p_{i}^{*(n)}, n=4$

$0 \quad 153494651842560$

$1 \quad 6395610493440$

$2 \quad 124448535691200$

$3 \quad 4968467473872$

$4 \quad 2749505046083$

i $\quad p_{i}^{*(n)}, n=5$

$0 \quad 1137902961267501753139200$

$1 \quad 47412623386145906380800$

$2 \quad 2067139141929439679969280$

$3 \quad 84522938940099251645760$

$4 \quad 343787425 \quad 2283265242 \quad 23240$

$5 \quad 11857916887903760599781$ 
Integer coefficients of the polynomial type Padé

approximants for $\Gamma(z)(n=1,2, \ldots, 10)$ (continued)

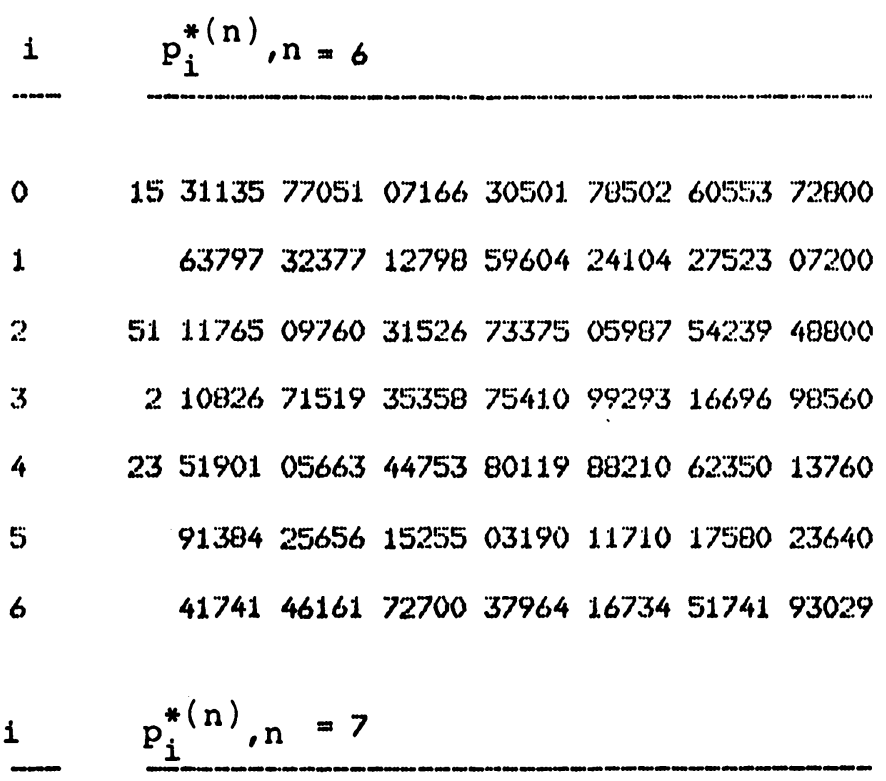

$0 \quad 129355290174041182710942331450483807$ 0183833464524800

1 $538980375725171594628926 \quad 3810436825$ 292451394355200

2

724460966839022651100774925295858508 143796609024000

3 30003094393654328891668543843755911 240865569525760

$4 \quad 73024432820 \quad 0754391373 \quad 305712522919149$ 026673782871040

5 $2945482808 \quad 45615897324173738826 \quad 08430$ 555954542915200 

$p_{i}^{*(n)}, n=8$ 604232359601539340713328640000

1382535287872337197175992315467791822 $650176348316730805863 \quad 3055360000$ $2859086633 \quad 35038 \quad 69498 \quad 180,30 \quad 20093 \quad 4551430141$ 911150953059729117098351001600

$11865975 \quad 1350454522 \quad 14782 \quad 2114106488948389$ 705036189009803281948788326400 $521428033196695 \quad 34421 \quad 16426 \quad 0333947621539965$ $40048 \quad 97355995883 \quad 02798 \quad 13395 \quad 45600$

$213 \quad 354262246626618 \quad 5542949596 \quad 1581259065$ 50903676641913702733735836483320

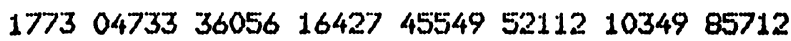
$692673641363684 \quad 423505926689920$

$67551677341593757 \quad 6484403333835547860485$ 029370060202234576349034766880 
Integer coefficients of the polynomial type Padé approximants for $\Gamma(z)(n=1,2, \ldots, 10)$ (continued)

$i \quad p_{i}^{*(n), n=9}$

0

$50 \quad 37574 \quad 4542479881717412185185422 \quad 15201$ 1174127602879095243299201983738140370994 685353656320000

1

209898935593666173822550771605925633

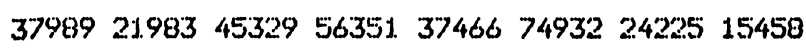
111889735680000

2

$636464474239650180583169668240268 \quad 35837$ 8704423098542291391582960987396613304724 352894238720000

$26 \quad 44817208853988693525937578769574617$ 0.200290297954098296281781041424920253781 4282706283640000 192515342692641918725703744498560812690 8664452661289732549005334636097024491908 309983468748800

$79 \quad 33551 \quad 3525281866 \quad 3512071010 \quad 68326 \quad 01384$

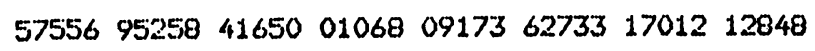
657759274291200

6 $14117482026924 \quad 1479297827957349090112156$ $90240242525684592962 \quad 87243 \quad 464097323806669$ 9036932.67077120

$56 \quad 3416759487 \quad 77673 \quad 38915229414298778206$

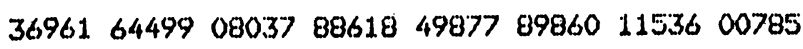
805624469018240 
Integer coefficients of the polynomial type Padé approximants for $\Gamma(z)(n=1,2, \ldots, 10)$ (continued)

$14797626 \quad 3534832957685183244398440074837$ 1206372278042632983670109606775835290346 439937904358760

477081430150669489354139658819546639 3705885971616137004168247823835686838860 942992541487979

i $p_{1}^{*(n)}, n=10$

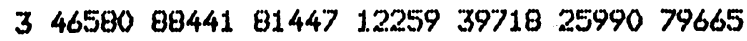
$7111482803783513255390681 \quad 1.7724 \quad 8805379079$

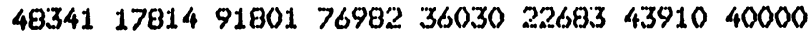
$144408701840893 \quad 63010808215941628319$ 4046311783490979718974611715718700224128 3118088242288250737426501259451432960000

$61 \quad 15426732424172079858982886771489551$ 0034884434194914768785851772853167898926 9497500123610834278127246823076659200000

254319727859810620993873499070236011 $6638540398 \quad 58186 \quad 48444803627 \quad 94045 \quad 3884407169$ 4567766225637666999312481942973186048000

28208654976682958180029765317442228462 1608672782329949267398896623467251261758 4137411674691183917394233772346048512000 3185307089488339449109329383956285384224 4155491812369343842975149420579491840000 
Integer coefficients of the polynomial type Padé approximants for $\Gamma(z)(n=1,2, \ldots, 10)$ (continued)

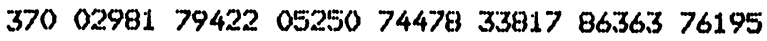
2268793218128552715122833766246724938042 $985451629950457679820278103622 \quad 3158743040$ $1504270 \quad 22336 \quad 604525914077817 \quad 1185477847$

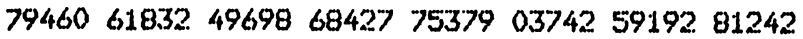
$777224026499963007636888469370 \quad 1461.288960$ 7616850444821407532976395054412442377573 9965053249408017228600048098585258420800 3863718237881242145702392221472834694 $1677980308 \quad 43903 \quad 3965564180293997317465049$ $87523752847031068830 \quad 02552000076349124808$ 10

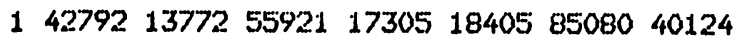
5366094330978887628173196048918628341663 6587676865006633701412048245295719812037 